\title{
Taking back control: a better deal for junior doctor engagement in rota design using e-Delphi and Assignment Problem Algorithm
}

\author{
Authors: Matthew Szeto* and Jasmine Mann
}

\section{Introduction}

The British Medical Association and NHS Employers published a guide to good rostering practice, which emphasised the need for a collaborative approach, in which the views of junior doctors should be taken into account when designing a rota. ${ }^{1}$ The guidance, however, did not provide tools for promoting junior doctor engagement in this process. Lack of structured consultation strategy and low level of satisfaction contribute to the prevailing narrative that junior doctor's input is undervalued, resulting in 'a crisis in junior doctor engagement'. ${ }^{2}$

This case study presents the utility of e-Delphi exercise for consultation and Assignment Problem Algorithm to improve satisfaction by matching work patterns to doctors' preferences.

\section{Methods}

Following review of workload and medical staffing, Medway Maritime Hospital undertook a redesign of its medical registrar (MR) rota in October 2018. With support from the Medical Staffing Department, a new rota was drafted by a doctor who takes part in the MR rota.

The draft rota, together with information on changes in work hours and pay, formed the basis of an e-Delphi consultation exercise.

> All MR were invited to participate in an online survey to rate statements regarding aspects of the new rota using a 5-point Likert scale, and to provide comments to justify their ratings.

> The quantitative and qualitative results from round 1 were summarised in a report to all MR. Having considered the report, all MR were then invited to reconsider the statements in round 2 . The process would be repeated for round 3 .

> A consensus was defined as statements being rated 'Agreed' or 'Strongly agreed' by $\geq 66 \%$ of respondents. ${ }^{3}$

> For statements that did not achieve a consensus, decisions would be taken based on simple majority.

The rota template was revised based on the result of the e-Delphi consultation. All MR were then invited to submit their top three

Authors: Medway NHS Foundation Trust ${ }^{*} \mathrm{RCP}$ chief registrar preferences for work pattern (ie which line of the rota they wish to occupy). This was constructed as an applied mathematics 'assignment problem'.

> The 'cost' of assigning each MR to their first preference was 1, their second preference was 2, and their third preference was 3. A cost of 99 was applied the rest of the rota.

> Using OpenSolver 2.9.0 (2018), an algorithm was used to populate the rota with the minimum 'total cost'. An example of such algorithm output is shown in Fig 1.

(A more detailed explanation is available at www.excel-easy.com/ examples/assignment-problem.html)

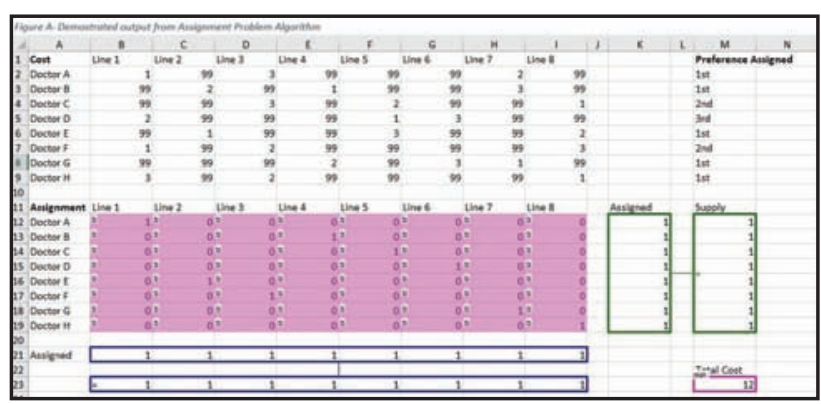

Fig 1. Demostrated output from Assignment Problem Algorithm

The process for designing and implementing the new MR rota was evaluated in another online survey 6 weeks post-implementation of the new rota.

\section{Results}

The return rate of the e-Delphi exercise was $72 \%$ for round 1 and $78 \%$ for round 2 . Round 3 was cancelled as there was no further qualitative responses to drive further consensus after round 2 . Four statements achieved consensus in round 1, and 2 additional statements achieved consensus in round 2 . There was a trend towards increasing agreement for all statements (Table 1).

Fourteen MRs submitted their work pattern preferences. All were allocated to one of their top three preferences as follow:

\footnotetext{
$>$ first preference: 4

$>$ second preference: 6

$>$ third preference: 4 .
} 


\section{Statements achieving consensus in round 1 (\% agreement)}

$>$ The new rota would allow for a better specialist registrar on-call experience. $(85 \%)$

$>$ The new rota would allow better patient care. $(85 \%)$

$>$ Not having to work two consecutive weekends is a desirable feature. $(77 \%)$

> If swaps cannot be found after reasonable effort, locum cover should be sought to honour existing leave arrangements. $(100 \%)$

Statements achieving consensus in round 2 (\% agreement in round $1, \%$ agreement in round 2 )

$>$ Existing agreed leaves should be honoured even if no swap or locum cover can be found. $(46 \%, 78 \%)$

> The new rota should be implemented from January 2019. $(63 \%, 71 \%)$

Statements accepted on simple majority (\% agreement in round $1, \%$ agreement in round 2 )

It is preferable to have an additional rest day AFTER (rather than before) weekend nights. (15\%, 57\%)

Provided the number of night shifts remains constant, spreading out night shift blocks is a desirable feature. $(54 \%$, $64 \%)$

Post-implementation evaluation (response rate: $89 \%$ ) showed high level of satisfaction with the design, allocation, and implementation of the new rota (Fig 2).

\section{Conclusion}

Good consultation strategy and high satisfaction level are facilitators for junior doctor engagement. ${ }^{4} \mathrm{E}$-Delphi exercise provides structured consultation to develop consensus for rota

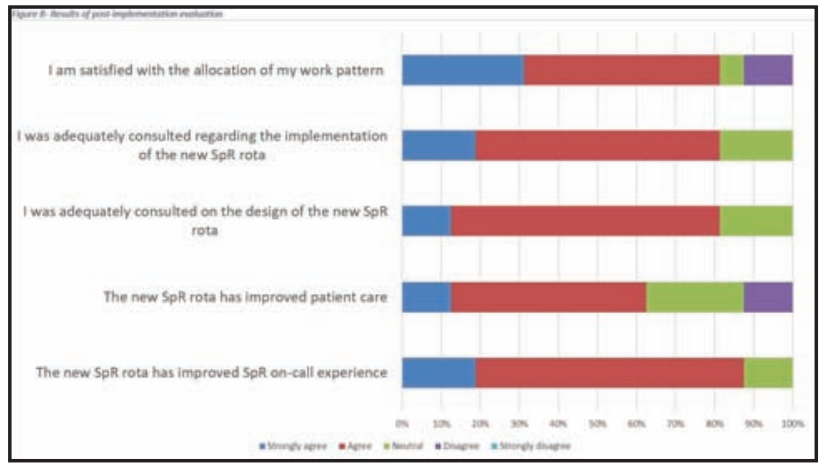

Fig 2. Results of post-implementation evaluation

design and implementation, while an Assignment Problem Algorithm increases satisfaction by matching work patterns to doctors' preferences. These tools should be considered in promoting engagement of junior doctors in rota design.

\section{References}

1 British Medical Association, NHS Employers. Good rostering guide. NHS and BMA, 2018. www.nhsemployers.org/case studies and resources/2018/05/good rostering guide [Accessed 24 February 2019].

2 Wathes R, Spurgeon P. Junior doctor engagement - investing in the future. London: Faculty of Medical Leadership and Management, 2016. www.fmlm.ac.uk/resources/junior-doctor-engagement\%E2 \% 80\% 93-investing-in-the-future [Accessed 24 February 2019].

3 Diamond IR, Grant RC, Feldman BM et al. Defining consensus: a systematic review recommends methodologic criteria for reporting of Delphi studies. J Clin Epidemiol 2014;67:401-9.

4 OpenSolver. OpenSolver for Excel. OpenSolver, 2018. https://opensolver. org/ [Accessed 24 February 2019]. 